\title{
Synthesis of new 4,6-disubstituted-1,3-oxazinan-2-one analogues
}

\author{
ARUN JYOTI BORAH and PRODEEP PHUKAN* \\ Department of Chemistry, Gauhati University, Guwahati 781 014, India \\ e-mail: pphukan@yahoo.com
}

MS received 2 August 2012; revised 17 August 2013; accepted 21 August 2013

\begin{abstract}
Oxazinan-2-one analogues are important heterocyclic compounds having significant biological activities. This study reports the synthesis of eight new 4,6-disubstituted -1,3-oxazinan-2-one analogues from corresponding homoallylic carbamates. Homoallylic carbamates were synthesized via a three-component reaction of aldehyde, allyltrimethylsilane and benzyl carbamate in presence of iodine as catalyst. In the next step, homoallylic carbamates were subjected to Sharpless dihydroxylation (racemic) to produce 3,4dihydroxybutylcarbamate derivatives. This product was then treated with $\mathrm{NaH}$ in tetrahydrofuran (THF) to produce the desired 6-(hydroxymethyl)-1,3-oxazinan-2-one in high yield.
\end{abstract}

Keywords. 1,3-Oxazinan-2-one; homoallylic carbamates; Sharpless dihydroxylation; iodine; heterocyclic compound.

\section{Introduction}

Heterocyclic compounds containing nitrogen and oxygen in the skeleton are important building blocks in the synthesis of biologically active compounds. ${ }^{1} 1,3-$ Oxazinan-2-one skeleton is one of such important compounds, used as building block for the synthesis of new heterocycles of biological significance. These 6memebered cyclic carbamates derivatives are found in many biologically important natural products such as maystansine, maystanprine, colubrinol and maytanbutine. ${ }^{2-4}$ 1, 3-Oxazinan-2-one derivatives exhibit a variety of biological activities such as antibacterial, ${ }^{5}$ antiinflammatory ${ }^{6}$ and antithrombotic ${ }^{7}$ properties. These molecules are used in treating asthma, allergies, ulcers, arthritis and diabetes. ${ }^{4 \mathrm{~b}}$ In addition, they are also being used as anticonvulsant, ${ }^{8}$ penetration enhancer, ${ }^{9}$ sedative, ${ }^{10}$ and analgesic. ${ }^{11}$ Some 6-phenyl-1, 3-oxazinan-2-one derivatives have phosphodiesterase IV inhibitor property and are used as remedies for inflammatory diseases and anti-asthmatics. ${ }^{12}$ 1,3-Oxazinan-2-one derivatives have also been used as key intermediates in the synthesis of several biologically important compounds such as L-ristosamine, L-daunosamine, ${ }^{13}( \pm)$ negamycin ${ }^{14}$ erythromycin $\mathrm{A}^{15}$ and thrombolytics. ${ }^{7}$ Besides being important bulding blocks in the preparation of complex synthetic targets, they are also used for the synthesis of amino alcohols ${ }^{16,17}$ and liquid crystal devices. ${ }^{18}$

*For correspondence
There are very limited approaches to the synthesis of 1, 3-oxazinan-2-one in literature. Lohray ${ }^{19}$ developed a method for enantiospecific synthesis of 6substituted $N$-aryl-1,3-oxazinan-2-ones using aspartic acid as starting material. A quicker synthesis of the same was carried out by reductive amination of 2deoxy-D-ribose followed by cyclization of aryl chloroformate derivatized amine. ${ }^{20}$ Other methods include halogen-mediated cyclization reactions, ${ }^{15 b, c, 21-30}$ rearrangement from cyclic sulphates, ${ }^{31}$ selenium-mediated cyclization of amino alcohols with carbon monoxide, ${ }^{32}$ asymmetric dihydroxylation of homoallylic amine, ${ }^{33}$ tethered aminohydroxylation, ${ }^{34}$ Hofmann rearrangement of primary amide ${ }^{35}$ and intramolecular Michael addition. ${ }^{36}$ Cyclization of 1, 3-aminoalcohol is one of the versatile methods in the synthesis of cyclic carbamate. ${ }^{20,37-40}$ A method used for the synthesis of oxazinanones via intramolecular diazocarbonyl insertion reaction of activated diazoketone catalysed by metal triflates was reported by Avery et al. ${ }^{41}$

\section{Experimental}

\subsection{Synthesis of homoallylic carbamate $(\boldsymbol{1})^{42}$}

Benzyl carbamate (2.55 mmol, 1.05 equiv) and allyltrimethylsilane $(2.5 \mathrm{mmol}, 1$ equiv) were added to a solution of aldehyde ( $2.5 \mathrm{mmol}, 1$ equiv) and iodine $(10 \mathrm{~mol} \%)$ in acetonitrile at room temperature. The reaction was completed within 10-20 min. After completion of the reaction thin layer chromatography 
(TLC), sodium thiosulphate was added to reaction mixture. The mixture was extracted with EtOAc. The organic extract was washed with brine and dried over anhydrous sodium sulphate. Evaporation of solvent and purification of the crude product using flash chromatography on silica gel (230-400 mesh) with petroleum ether-EtOAc (10-20\%) as eluent gave the pure product (yield 50-82\%).

2.1a Benzyl 1-(4-chlorophenyl)but-3-enylcarbamate 1a: Yield: $70 \%$ (colourless solid); mp: $68-70^{\circ} \mathrm{C}$; IR $\left(\mathrm{KBr}, \mathrm{cm}^{-1}\right): 3342,3064,2950,1678,1534,1489$, $1346,1263,1119,1035,752 ;{ }^{1} \mathrm{H}$ NMR $\left(\mathrm{CDCl}_{3}\right.$, $300 \mathrm{MHz}): \delta 7.35-7.19(\mathrm{~m}, 9 \mathrm{H}), 5.72-5.57(\mathrm{~m}, 1 \mathrm{H})$, 5.15-5.02 (m, 5H), $4.71(\mathrm{br}, 1 \mathrm{H}), 2.50(\mathrm{br}, 2 \mathrm{H}) ;{ }^{13} \mathrm{C}$ NMR $\left(\mathrm{CDCl}_{3}, 75 \mathrm{MHz}\right): \delta 155.6,140.5,136.2,133.2$, $133.0,128.7,128.5,128.2,127.6,118.9,66.9,53.9$, 40.9 .

2.1b Benzyl 1-(4-bromophenyl)but-3-enylcarbamate 1b: Yield: $75 \%$ (colourless solid); mp: $87-88^{\circ} \mathrm{C}$; IR $\left(\mathrm{KBr}, \mathrm{cm}^{-1}\right): 3345,3087,2950,1684,1592,1533$, $1455,1347,1264,1119,1034,751 ;{ }^{1} \mathrm{H}$ NMR $\left(\mathrm{CDCl}_{3}\right.$, $400 \mathrm{MHz}): \delta 7.44(\mathrm{~d}, J=8.1 \mathrm{~Hz}, 2 \mathrm{H}), 7.40-7.25$ $(\mathrm{m}, 5 \mathrm{H}), 7.13(\mathrm{~d}, J=7.7 \mathrm{~Hz}, 2 \mathrm{H}), 5.72-5.54(\mathrm{~m}$, $1 \mathrm{H}), 5.22-4.96(\mathrm{~m}, 5 \mathrm{H}), 4.82-4.65(\mathrm{~m}, 1 \mathrm{H}), 2.60-2.36$ $(\mathrm{m}, 2 \mathrm{H}) ;{ }^{13} \mathrm{C} \mathrm{NMR}\left(\mathrm{CDCl}_{3}, 100 \mathrm{MHz}\right): \delta 155.6,141.0$, $136.2,133.2,131.6,128.5,128.2,127.9,121.1,118.9$, $66.9,53.9,40.8$.

2.1c Benzyl 1-p-tolylbut-3-enylcarbamate 1c: Yield: 72\% (colourless solid); mp: $70-71^{\circ} \mathrm{C}$; IR $\left(\mathrm{KBr}, \mathrm{cm}^{-1}\right)$ : 3357, 3058, 2958, 1692, 1590, 1526, 1457, 1350, 1263, $1143,1044,732 ;{ }^{1} \mathrm{H}$ NMR $\left(\mathrm{CDCl}_{3}, 300 \mathrm{MHz}\right): \delta 7.35-$ $7.16(\mathrm{~m}, 9 \mathrm{H}), 5.75-5.62(\mathrm{~m}, 1 \mathrm{H}), 5.14-5.07(\mathrm{~m}, 5 \mathrm{H})$, $4.78(\mathrm{~m}, 1 \mathrm{H}), 2.56-2.62(\mathrm{~m}, 2 \mathrm{H}), 2.34(\mathrm{~s}, 3 \mathrm{H}) ;{ }^{13} \mathrm{C}$ NMR $\left(\mathrm{CDCl}_{3}, 75 \mathrm{MHz}\right): \delta 155.6,138.8,136.9,136.4$, $133.8,129.2$, 128.4, 128.1, 126.1, 118.2, 66.6, 54.2, $40.9,30.9$.

2.1d Benzyl 1-(4-methoxyphenyl)but-3-enylcarbamate 1d: Yield: $75 \%$ (colourless solid); mp: $70-71{ }^{\circ} \mathrm{C}$; IR (KBr, $\mathrm{cm}^{-1}$ ): 3362, 3067, 2950, 1685, 1597, 1528, 1454, 1346, 1243, 1116, 1032, 748; ${ }^{1} \mathrm{H}$ NMR $\left(\mathrm{CDCl}_{3}\right.$, $400 \mathrm{MHz}): \delta 7.41-7.29(\mathrm{~m}, 5 \mathrm{H}), 7.19(\mathrm{~d}, J=7.8 \mathrm{~Hz}$, $2 \mathrm{H}), 6.86(\mathrm{~d}, J=8.6 \mathrm{~Hz}, 2 \mathrm{H}), 5.77-5.58(\mathrm{~m}, 1 \mathrm{H})$, 5.20-5.00 (m, 5H), 4.80-4.70 (m, 1H), $3.79(\mathrm{~s}, 3 \mathrm{H})$, 2.60-2.54 (m, 2H); ${ }^{13} \mathrm{C}$ NMR $\left(\mathrm{CDCl}_{3}, 100 \mathrm{MHz}\right): \delta$ $158.7,155.6,136.4,133.9,128.4,128.0,127.3,118.2$, $113.8,86.7,55.2,53.9,40.9$. 2.1e Benzyl 1-(naphthalene-6-yl)but-3-enylcarbamate 1e: Yield: $65 \%$ (colourless solid); $\mathrm{mp}$ : $73-75^{\circ} \mathrm{C}$; IR ( $\mathrm{KBr}$, $\left.\mathrm{cm}^{-1}\right)$ : 3365, 3049, 2970, 1686, 1528, 1323, 1250, $1122,1036,751 ;{ }^{1} \mathrm{H} \mathrm{NMR}\left(\mathrm{CDCl}_{3}, 300 \mathrm{MHz}\right): \delta 7.86-$ $7.27(\mathrm{~m}, 12 \mathrm{H}), 5.79-5.65(\mathrm{~m}, 1 \mathrm{H}), 5.30(\mathrm{~m}, 1 \mathrm{H})$, 5.18-5.01 (m, 5H), $2.65(\mathrm{br}, 2 \mathrm{H}) ;{ }^{13} \mathrm{C} \mathrm{NMR}\left(\mathrm{CDCl}_{3}\right.$, $75 \mathrm{MHz}): \delta 155.6,139.2,136.3,133.6,133.2,132.7$, $128.4,128.4,128.1,127.8,127.5,126.1,125.7,124.8$, $124.4,118.5,66.8,54.5,40.9$.

2.1f Benzyl 1-(4-flurophenyl)but-3-enylcarbamate 1f: Yield: $71 \%$ (yellowish liquid); IR $\left(\mathrm{KBr}, \mathrm{cm}^{-1}\right): 3445$, 1697, 1604, 1540, 1349, 1224, 1159, 1041, 736; ${ }^{1} \mathrm{H}$ NMR $\left(\mathrm{CDCl}_{3}, 400 \mathrm{MHz}\right): \delta 7.33-7.00(\mathrm{~m}, 9 \mathrm{H}), 5.66-$ $5.63(\mathrm{~m}, 1 \mathrm{H}), 5.12-5.05(\mathrm{~m}, 5 \mathrm{H}), 4.77(\mathrm{br}, 1 \mathrm{H}), 2.50(\mathrm{br}$, $\mathrm{s}, 2 \mathrm{H}) ;{ }^{13} \mathrm{C}$ NMR $\left(\mathrm{CDCl}_{3}, 100 \mathrm{MHz}\right): \delta 163.1,160.7$, 155.6, 137.1, 136.3, 133.4, 128.5, 128.1, 127.8, 127.8, 118.7, 115.4, 115.2, 66.8, 53.8, 41.3.

2.1g Benzyl (1-phenylhex-5-en-3-yl)carbamate 1g: Yield: $70 \%$ (colourless solid); mp: $70-71{ }^{\circ} \mathrm{C}$; IR (Nujol, $\left.\mathrm{cm}^{-1}\right): 3065,1696,1534,1451,1243,1045,739 ;{ }^{1} \mathrm{H}$ NMR $\left(\mathrm{CDCl}_{3}, 400 \mathrm{MHz}\right): \delta 7.32-7.16(\mathrm{~m}, 7 \mathrm{H}), 7.13-$ $7.06(\mathrm{~m}, 3 \mathrm{H}), 5.77-5.60(\mathrm{~m}, 1 \mathrm{H}), 5.10-4.95(\mathrm{~m}, 4 \mathrm{H})$, $4.54(\mathrm{~d}, J=7.8 \mathrm{~Hz}, 1 \mathrm{H}), 3.79-3.63(\mathrm{~m}, 1 \mathrm{H}), 2.68-$ $2.50(\mathrm{~m}, 2 \mathrm{H}), 2.29-2.09(\mathrm{~m}, 2 \mathrm{H}), 1.83-1.70(\mathrm{~m}, 1 \mathrm{H})$; ${ }^{13} \mathrm{C} \mathrm{NMR}\left(\mathrm{CDCl}_{3}, 100 \mathrm{MHz}\right): \delta 156.2,141.7,136.6$, $133.9,128.5,128.4,128.3,128.1,125.9,118.1,66.6$, $50.5,39.5,36.5,32.3$.

2.1h Benzyl 1-(2-methoxyphenyl)but-3-enylcarbamate $\mathbf{l h}$ : Yield: $80 \%$ (colourless solid); mp: $88-90^{\circ} \mathrm{C}$; IR $\left(\mathrm{KBr}, \mathrm{cm}^{-1}\right)$ : 3333, 3060, 2942, 1685, 1600, 1537, $1468,1352,1264,1121,1035,754 ;{ }^{1} \mathrm{H}$ NMR $\left(\mathrm{CDCl}_{3}\right.$, $400 \mathrm{MHz}): \delta 7.30-6.83(\mathrm{~m}, 9 \mathrm{H}), 5.63-5.61(\mathrm{~m}, 2 \mathrm{H})$, 5.04-4.98 (m, 5H), 3.80 (s, 3H), 2.52 ( br, s, $2 \mathrm{H}) ;{ }^{13} \mathrm{C}$ NMR $\left(\mathrm{CDCl}_{3}, 100 \mathrm{MHz}\right): \delta 156.8,155.6,136.5,134.7$, $129.3,128.4,128.3,128.2,128.1,120.5,117.4,110.8$, $66.6,55.2,52.8,39.7$.

\subsection{Synthesis of 3,4-dihydroxybutylcarbamate derivative $(2)^{43}$}

A flask was charged with $\mathrm{K}_{2} \mathrm{CO}_{3}(4.5 \mathrm{mmol})$ and $\mathrm{K}_{3} \mathrm{Fe}(\mathrm{CN})_{6}(4.5 \mathrm{mmol})$ in a mixture of ${ }^{t} \mathrm{BuOH}$ and water (1:1). The mixture was stirred for 5-10 min and a drop of pyridine and $\mathrm{K}_{2} \mathrm{OsO}_{2}(\mathrm{OH})_{4}(0.006 \mathrm{mmol})$ was added to the mixture and stirred for another $5 \mathrm{~min}$. Thereafter, homoalylic carbamate, $1(1.5 \mathrm{mmol})$ was added to the reaction mixture and stirred for $24 \mathrm{~h}$. The reaction was then quenched with sodium sulphite and 
the reaction mixture was stirred for another half an hour. The reaction mixture was then extracted with EtOAc. The organic extract was washed with brine and dried over anhydrous sodium sulphate. The solvent was evaporated under reduced pressure to get the crude mixture. The crude product was purified by flash column chromatography using petroleum ether and ethyl acetate (1:1) as eluent to get the desired diol as a inseparable mixture of two diastereomers (yield: 70-95\%).

2.2a Benzyl 1-(4-chlorophenyl)-3, 4-dihydroxybutylcarbamate 2a: Yield: $72 \%$ (colourless solid); mp: 103-105 ${ }^{\circ} \mathrm{C}$; IR $\left(\mathrm{KBr}, \mathrm{cm}^{-1}\right)$ : 3429 (br), 2930, 1684, 1593, 1539, 1454, 1350, 1261, 1094, 1013,758; ${ }^{1} \mathrm{H}$ NMR $\left(\mathrm{CDCl}_{3}, 400 \mathrm{MHz}\right): \delta 7.27-7.13(\mathrm{~m}, 18 \mathrm{H}), 5.54$ $(\mathrm{d}, J=8 \mathrm{~Hz}, 2 \mathrm{H}), 5.06-4.94(\mathrm{~m}, 4 \mathrm{H}), 3.68(\mathrm{br}$, s, 2H), 3.56-3.48 (m, 2H), 3.42-3.31 (m, 2H), 2.36 (br, s, 2H), 1.84-1.75 (m, 2H), 1.68-1.63 (m, 2H); ${ }^{13} \mathrm{C}$ NMR $\left(\mathrm{CDCl}_{3}, 75 \mathrm{MHz}\right): \delta 156.6,155.9,140.1$, $136.1,135.9,133.1,128.8,128.5,128.4,128.2,128.1$, 127.9, 127.7, 127.6, 69.8, 69.8, 68.6, 67.1, 66.9, 66.4, 51.6, 39.5, 39.2; MS $(m / z): 372.1\left(\mathrm{M}^{+}+\mathrm{Na}\right), 373.1$; Anal. calcd. for $\mathrm{C}_{18} \mathrm{H}_{20} \mathrm{ClNO}_{4}(\%)$ : C, 61.80; H, 5.76; N, 4.00; Found: C, 61.75; H, 5.90; N, 4.10.

2.2b Benzyl 1-(4-bromophenyl)-3, 4-dihydroxybutylcarbamate $2 \boldsymbol{b}$ : Yield: $74 \%$ (colourless solid); mp: 123-125 ${ }^{\circ} \mathrm{C}$; IR $\left(\mathrm{KBr}, \mathrm{cm}^{-1}\right)$ : 3362, 3058, 2950, 1686, $1593,1525,1455,1346,1269,1097,1049,754 ;{ }^{1} \mathrm{H}$ NMR $\left(\mathrm{CDCl}_{3}, 400 \mathrm{MHz}\right): \delta 7.41-7.09(\mathrm{~m}, 18 \mathrm{H}), 5.52$ $5.46(\mathrm{~m}, 2 \mathrm{H}), 5.07-4.91(\mathrm{~m}, 4 \mathrm{H}), 3.69$ (br, 1H), 3.57$3.51(\mathrm{~m}, 3 \mathrm{H}), 3.44-3.32(\mathrm{~m}, 2 \mathrm{H}), 2.07-2.04(\mathrm{~m}, 1 \mathrm{H})$, 1.95-1.93 (m, 1H), 1.86-1.77 (m, 2H), 1.70-1.59 (m, $2 \mathrm{H}) ;{ }^{13} \mathrm{C} \mathrm{NMR}\left(\mathrm{CDCl}_{3}, 75 \mathrm{MHz}\right): \delta 156.7,155.8,140.3$, $136.1,135.9,131.8,131.8,128.5,128.5,128.3,128.2$, $128.1,127.9,124.3,121.4,121.3,69.9,68.5,67.2$, $66.9,66.5,66.4,53.5,51.7,39.5,39.2 ; \mathrm{MS}(\mathrm{m} / \mathrm{z})$ : $416.1\left(\mathrm{M}^{+}+\mathrm{Na}\right), 418.0\left(\mathrm{M}^{+}+2+\mathrm{Na}\right)$; Anal. calcd. for $\mathrm{C}_{18} \mathrm{H}_{20} \mathrm{BrNO}_{4}(\%)$ : C, 54.84; H, 5.11; N, 3.55; Found: C, 54.90; H, 5.15; N, 3.55.

2.2c Benzyl 3, 4-dihydroxy-1-p-tolylbutylcarbamate $2 c$ : Yield: $84 \%$ (colourless solid); mp: $102-104^{\circ} \mathrm{C}$; IR $\left(\mathrm{KBr}, \mathrm{cm}^{-1}\right)$ : 3345, 3283, 3055, 2928, 1697, 1538, $1456,1350,1257,1100,1014,737 ;{ }^{1} \mathrm{H}$ NMR $\left(\mathrm{CDCl}_{3}\right.$, $400 \mathrm{MHz}): \delta 7.57-7.35(\mathrm{~m}, 18 \mathrm{H}), 5.68-5.59(\mathrm{~m}, 2 \mathrm{H})$, 5.33-5.18 (m, 4H), 4.05-4.00 (m, 1H), 3.89-3.78 (m, $3 \mathrm{H}), 3.74-3.61$ (m, 2H), 2.82 (br, s, 2H), 2.55 (s, 6H), 2.20-1.95 (m, 4H); ${ }^{13} \mathrm{C}$ NMR $\left(\mathrm{CDCl}_{3}, 75 \mathrm{MHz}\right): \delta$ $156.8,155.9,138.4,137.2,136.2,135.9,129.4,128.4$, $128.4,128.2,128.1,126.2,126.1,69.9,68.7,67.1,66.7$,
66.5, 66.4, 53.3, 51.8, 39.7, 39.7, 21.1, 20.9; $\mathrm{MS}(\mathrm{m} / \mathrm{z})$ : 338.2 (8) $\left(\mathrm{M}^{+}+\mathrm{Na}\right), 339.0$ (15), 179.5 (55); Anal. calcd. for $\mathrm{C}_{19} \mathrm{H}_{23} \mathrm{NO}_{4}(\%)$ : C, 69.28; H, 7.04; N, 4.25; Found: C, 69.18; H, 6.95; N, 4.20.

2.2d Benzyl-3, 4-dihydroxy-1-(4-methoxyphenyl)butylcarbamate $2 d$ : Yield: $75 \%$ (colourless solid); mp: 67$70^{\circ} \mathrm{C}$; IR $\left(\mathrm{KBr}, \mathrm{cm}^{-1}\right): 3421,3352,3038,2940,1690$, 1606, 1528, 1459, 1346, 1243, 1096, 1047, 753; ${ }^{1} \mathrm{H}$ $\mathrm{NMR}\left(\mathrm{CDCl}_{3}, 400 \mathrm{MHz}\right): \delta 7.27-6.79(\mathrm{~m}, 18 \mathrm{H}), 5.22$ (m, 2H), 5.07-4.94 (m, 4H), 4.90-4.85 (m, 1H), 4.77$4.67(\mathrm{~m}, 1 \mathrm{H}), 3.72(\mathrm{~s}, 6 \mathrm{H}), 3.57-3.50(\mathrm{~m}, 2 \mathrm{H}), 3.46$ 3.33 (m, 2H), 2.10-2.08 (br, 2H), 1.92-1.66 (m, 4H); ${ }^{13} \mathrm{C}$ NMR $\left(\mathrm{CDCl}_{3}, 75 \mathrm{MHz}\right): \delta 158.9,156.9,156.1$, 136.2, 136.1, 133.4, 128.5, 128.4, 128.2, 128.1, 127.9, 127.6, 127.4, 114.1, 69.9, 68.7, 67.1, 66.8, 66.5, 66.4, 55.2, 55.2, 51.5, 51.5, 39.7; MS $(m / z): 368.1\left(\mathrm{M}^{+}+\right.$ $\mathrm{Na}, 100), 369.1$ (15); Anal. calcd. for $\mathrm{C}_{19} \mathrm{H}_{23} \mathrm{NO}_{5}(\%)$ : C, 66.07; H, 6.71; N, 4.06; Found: C, 66.10; H, 6.80; $\mathrm{N}, 4.10$.

2.2e Benzyl-3, 4-dihydroxy-1-(naphthalene-6-yl)butylcarbamate $2 e$ : Yield: 93\% (colourless solid); mp: 87$89^{\circ} \mathrm{C}$; IR $\left(\mathrm{KBr}, \mathrm{cm}^{-1}\right)$ : 3336, 3054, 2944, 1684, 1601, 1631, 1455, 1331, 1258, 1097, 1045, 742; ${ }^{1} \mathrm{H}$ NMR $\left(\mathrm{CDCl}_{3}, 400 \mathrm{MHz}\right): \delta 7.78-7.27(\mathrm{~m}, 24 \mathrm{H}), 5.75(\mathrm{~d}, J=$ $8 \mathrm{~Hz}, 2 \mathrm{H}), 5.17-5.01(\mathrm{~m}, 4 \mathrm{H}), 4.09-4.04(\mathrm{~m}, 1 \mathrm{H}), 3.77$ (br, 1H), 3.52-3.46 (m, 4H), 2.96-2.80 (m, 2H), 2.15$1.81(\mathrm{~m}, 4 \mathrm{H}) ;{ }^{13} \mathrm{C} \mathrm{NMR}\left(\mathrm{CDCl}_{3}, 100 \mathrm{MHz}\right): \delta 156.9$, $156.1,133.2,128.6,128.5,128.4,128,2,128.1,127.6$, 126.2, 67.2, 66.9, 66.6, 66.5, 53.8, 52.3, 39.7, 39.4, 30.9; $\mathrm{MS}(\mathrm{m} / \mathrm{z}): 388.2\left(\mathrm{M}^{+}+\mathrm{Na}, 39\right), 372.2(8)$; Anal. calcd. for $\mathrm{C}_{22} \mathrm{H}_{23} \mathrm{NO}_{4}(\%)$ : C, 72.31; H, 6.34; N, 3.83; Found: C, 72.25; H, 6.30; N, 3.87 .

2.2f Benzyl 1-(4-flurophenyl)-3, 4-dihydroxybutylcarbamate 2f: Yield: 95\% (colourless solid); mp: 79$82^{\circ} \mathrm{C}$; IR $\left(\mathrm{KBr}, \mathrm{cm}^{-1}\right): 3445,2925,1697,1593,1350$, $1226,1054,735 ;{ }^{1} \mathrm{H}$ NMR $\left(\mathrm{CDCl}_{3}, 400 \mathrm{MHz}\right): \delta 7.28$ $6.93(\mathrm{~m}, 18 \mathrm{H}), 5.43-5.34(\mathrm{~m}, 2 \mathrm{H}), 5.04-4.94(\mathrm{~m}, 4 \mathrm{H})$, $4.76(\mathrm{br}, 1 \mathrm{H}), 3.71$ (br, s, 1H), 3.59-3.52 (m, 2H), 3.45$3.34(\mathrm{~m}, 2 \mathrm{H}), 2.00-1.66(\mathrm{~m}, 6 \mathrm{H}) ;{ }^{13} \mathrm{C} \mathrm{NMR}\left(\mathrm{CDCl}_{3}\right.$, $75 \mathrm{MHz}): \delta 163.6,160.3,156.7,155.9,137.7,137.2$, $136.1,135.9,128.5,128.4,128.3,128.2,128.1,128.0$, 127.9, 115.7, 115.4, 69.9, 68.6, 67.2, 66.9, 66.5, 66.4, 53.1, 51.5, 39.7, 39.5 MS $(m / z): 356.1\left(\mathrm{M}^{+}+\mathrm{Na}, 100\right)$, 338.2 (10); Anal. calcd. for $\mathrm{C}_{18} \mathrm{H}_{20} \mathrm{FNO}_{4}(\%)$ : C, 64.85; H, 6.05; N, 4.20; Found: C, 64.80; H, 6.10; N, 4.25.

2.2g Benzyl (5,6-dihydroxy-1-phenylhexan-3-yl)carbamate 2g: Yield: 75\% (colourless solid); mp: 73$76^{\circ} \mathrm{C}$; IR $\left(\mathrm{KBr}, \mathrm{cm}^{-1}\right)$ : 3426, 3335, 2924, 1687, 1599 , 
$1541,1454,1352,1253,1051,750 ;{ }^{1} \mathrm{H} \mathrm{NMR}\left(\mathrm{CDCl}_{3}\right.$, $400 \mathrm{MHz}): \delta$ 7.32-7.05 (m, $20 \mathrm{H}), 5.06-5.04(\mathrm{~m}, 4 \mathrm{H})$, $4.74(\mathrm{br}, 1 \mathrm{H}), 4.61(\mathrm{~d}, J=12 \mathrm{~Hz}, 1 \mathrm{H}), 3.86-3.68(\mathrm{~m}$, $4 \mathrm{H}), 3.59-3.57(\mathrm{~m}, 1 \mathrm{H}), 3.51-3.48(\mathrm{~m}, 1 \mathrm{H}), 3.42-3.38$ (m, 2H), 2.70-2.55 (m, 4H), 1.81-1.64 (m, 4H), 1.60$1.51(\mathrm{~m}, 2 \mathrm{H}), 1.27-1.19(\mathrm{~m}, 2 \mathrm{H}) ;{ }^{13} \mathrm{C} \mathrm{NMR}\left(\mathrm{CDCl}_{3}\right.$, $75 \mathrm{MHz}): \delta 157.6,156.5,141.3,141.1,136.3,136.1$, $128.6,128.5,128.5,128.4,128.3,128.2,128.1,128.0$, 127.9, 126.0, 125.9, 70.1, 68.3, 67.2, 66.8, 66.5, 66.4, 49.3, 47.9, 39.8, 38.9, 37.5, 37.4, 32.6, 32.1; MS ( $\mathrm{m} / \mathrm{z})$ : $366.0\left(\mathrm{M}^{+}+\mathrm{Na}\right)$; Anal. calcd. for $\mathrm{C}_{20} \mathrm{H}_{25} \mathrm{NO}_{4}(\%)$ : $\mathrm{C}$, 69.95; H, 7.34; N, 4.08; Found: C, 69.90; H, 7.30; N, 4.12 .

2.2h Benzyl-3,4-dihydroxy-1-(2-methoxyphenyl)butylcarbamate $2 \mathrm{~h}$ : Yield: $70 \%$ (colourless solid); mp: $110-113^{\circ} \mathrm{C}$; IR $\left(\mathrm{KBr}, \mathrm{cm}^{-1}\right)$ : 3430, 3338, 2927, 1686, $1597,1543,1469,1351,1259,1101,1051,755 ;{ }^{1} \mathrm{H}$ NMR $\left(\mathrm{CDCl}_{3}, 400 \mathrm{MHz}\right): \delta 7.30-6.81(\mathrm{~m}, 18 \mathrm{H}), 5.98$ $(\mathrm{d}, J=8 \mathrm{~Hz}, 1 \mathrm{H}), 5.81(\mathrm{br}, \mathrm{s}, 1 \mathrm{H}), 5.04-4.96(\mathrm{~m}, 6 \mathrm{H})$, $3.78(\mathrm{~s}, 6 \mathrm{H}), 3.53-3.50(\mathrm{~m}, 2 \mathrm{H}), 3.44-3.37(\mathrm{~m}, 2 \mathrm{H})$, 2.25 (br, 2H), 1.93-1.81 (m, 3H), 1.61-1.54 (m, 1H); ${ }^{13} \mathrm{C} \mathrm{NMR}\left(\mathrm{CDCl}_{3}, 100 \mathrm{MHz}\right): \delta 157.4,156.9,156.7$, 156.0, 136.4, 136.2, 128.9, 128.8, 128.7, 128.6, 128.5, 128.3, 128.1, 121.1, 120.9, 111.1, 111.0, 70.2, 68.7, 67.2, 66.8, 66.7, 66.4, 55.4, 55.3, 51.3, 51.1, 39.3, 39.0; MS $(m / z): 368.1\left(\mathrm{M}^{+}+\mathrm{Na}, 100\right), 369.1\left(\mathrm{M}^{+}+1+\mathrm{Na}\right.$ ,18); Anal. calcd. for $\mathrm{C}_{19} \mathrm{H}_{23} \mathrm{NO}_{5}(\%)$ : C, 66.07; H, 6.71; N, 4.06; Found: C, 66.10; H, 6.75; N, 4.10.

\subsection{Synthesis of 1, 3-oxazinan-2-one $(3)^{44}$}

The diol (2, $1 \mathrm{mmol}, 1$ equiv) was dissolved in dry THF (3-4 mL) under $\mathrm{N}_{2}$ atmosphere. The mixture was then cooled in an ice water bath and $\mathrm{NaH}(1.4 \mathrm{mmol}$, 1.4 equiv) was added to the mixture. The reaction mixture was then allowed to stir at $0-5^{\circ} \mathrm{C}$ for appropriate time (TLC). The reaction was then quenched with aq. $\mathrm{NH}_{4} \mathrm{Cl}$ solution and extracted with EtOAc. The organic extract was then dried over anhydrous sodium sulphate. The solvent was evaporated under reduced pressure to get the crude reaction mixture. The crude product was purified by flash column chromatography to get the 1, 3-oxazinan-2-one as a inseparable diastereomeric mixture (yield 70-85\%).

2.3a 4-(4-Chlorophenyl)-6-(hydroxymethyl)-1, 3-oxazinan2-one $(3 \boldsymbol{a}+4 \boldsymbol{a})$ : Yield: $75 \%$ (colourless solid); mp: $158-160^{\circ} \mathrm{C}$; IR $\left(\mathrm{KBr}, \mathrm{cm}^{-1}\right)$ : $3515,3283,3058,2938$, $1714,1541,1492,1340,1291,1087,1056,766 ;{ }^{1} \mathrm{H}$ NMR (DMSO, $400 \mathrm{MHz}) \delta 7.73$ (br, m, 1H), 7.61 (br, s, 1H), 7.46-7.42 (m, 4H), 7.36-7.33 (m, 4H), 5.00 $4.93(\mathrm{~m}, 2 \mathrm{H}), 4.70-4.69(\mathrm{~m}, 1 \mathrm{H}), 4.60(\mathrm{dd}, J=12 \mathrm{~Hz}$, $4 \mathrm{~Hz}, 1 \mathrm{H}), 4.34-4.30(\mathrm{~m}, 1 \mathrm{H}), 3.97-3.92(\mathrm{~m}, 1 \mathrm{H}), 3.52-$ $3.44(\mathrm{~m}, 2 \mathrm{H}), 2.13-2.05(\mathrm{~m}, 2 \mathrm{H}), 1.89-1.86(\mathrm{br}, \mathrm{m}$, 1H), 1.59-1.50 (m, 1H); ${ }^{13} \mathrm{C}$ NMR (DMSO, $100 \mathrm{MHz}$ ): $\delta 153.2,152.9,142.3,141.3,132.0,131.7,128.5,128.4$, 128.1, 128.0, 76.7, 73.4, 62.8, 62.7, 53.1, 50.5, 32.9, 30.4; $\mathrm{MS}(\mathrm{m} / \mathrm{z}): 264.1\left(\mathrm{M}^{+}+\mathrm{Na}\right), 266.1\left(\mathrm{M}^{+}+2+\mathrm{Na}\right)$; Anal. calcd. for $\mathrm{C}_{11} \mathrm{H}_{12} \mathrm{ClNO}_{3}(\%)$ : C, 54.67; $\mathrm{H}, 5.00 ; \mathrm{N}$, 5.80; Found: C, 54.60; H, 4.95; N, 5.70.

$2.3 \mathrm{~b}$ 4-(4-Bromophenyl)-6-(hydroxymethyl)-1, 3-oxazinan-2-one $(3 \boldsymbol{b}+\mathbf{4 b})$ : Yield: $82 \%$ (colourless solid); $\mathrm{mp}: 173-175^{\circ} \mathrm{C}$; IR $\left(\mathrm{KBr}, \mathrm{cm}^{-1}\right)$ : 3418, 3293, 3126, 2936, 1710, 1592, 1538, 1459, 1341, 1292, 1086, 1057, $768 ;{ }^{1} \mathrm{H}$ NMR (DMSO, $\left.400 \mathrm{MHz}\right): \delta 7.72(\mathrm{~d}, J=$ $4 \mathrm{~Hz}, 1 \mathrm{H}), 7.61-7.55$ (m, 5H), 7.29-7.26 (m, 4H), 4.98$4.94(\mathrm{~m}, 2 \mathrm{H}), 4.68-4.66(\mathrm{~m}, 1 \mathrm{H}), 4.58(\mathrm{dd}, J=$ $12 \mathrm{~Hz}, 4 \mathrm{~Hz}, 1 \mathrm{H}), 4.33-4.30(\mathrm{~m}, 1 \mathrm{H}), 3.96-3.92(\mathrm{~m}$, $1 \mathrm{H}), 3.50-3.45(\mathrm{~m}, 2 \mathrm{H}), 2.12-2.04(\mathrm{~m}, 2 \mathrm{H}), 1.88-1.85$ (br, m, 1H), 1.58-1.49 (m, 1H); ${ }^{13} \mathrm{C}$ NMR (DMSO, $100 \mathrm{MHz}): \delta 153.2,152.9,142.7,141.7,131.4,131.3$, $128.5,128.3,120.5,120.2,76.7,73.3,62.8,62.7,53.2$, 50.6, 32.9, 30.3; MS $(m / z): 308.0\left(\mathrm{M}^{+}+\mathrm{Na}\right), 310.0$ $\left(\mathrm{M}^{+}+2+\mathrm{Na}\right)$; Anal. calcd. for $\mathrm{C}_{11} \mathrm{H}_{12} \mathrm{BrNO}_{3}(\%)$ : $\mathrm{C}$, 46.18; H, 4.23; N, 4.90; Found: C, 46.25; H, 4.30; $\mathrm{N}, 4.85$.

2.3c 6-(Hydroxymethyl)-4-p-tolyl-1, 3-oxazinan-2-one $(3 c+4 c)$ : Yield: $73 \%$ (colourless solid); mp: 124$125^{\circ} \mathrm{C}$; IR $\left(\mathrm{KBr}, \mathrm{cm}^{-1}\right): 3372,3272,2969,2917,1707$, 1592, 1538, 1464, 1353, 1294, 1090, 1052; ${ }^{1} \mathrm{H}$ NMR (DMSO, $400 \mathrm{MHz}): \delta 7.66(\mathrm{~m}, 1 \mathrm{H}), 7.50(\mathrm{br}, \mathrm{s}, 1 \mathrm{H})$, 7.20-7.15 (m, 8H), 4.97-4.91 (m, 2H), $4.63(\mathrm{~m}, 1 \mathrm{H})$, $4.52(\mathrm{dd}, J=12 \mathrm{~Hz}, 4 \mathrm{~Hz}, 1 \mathrm{H}), 4.32-4.29(\mathrm{~m}, 1 \mathrm{H})$, 3.96-3.94 (m, 1H), 3.50-3.43 (m, 2H), 2.29 (s, 6H), 2.08-2.02 (m, 2H), 1.86-1.83 (m, 1H), 1.59-1.49 (m, $1 \mathrm{H}) ;{ }^{13} \mathrm{C}$ NMR (DMSO,100 MHz): $\delta$ 153.5, 153.3, $140.3,139.3,136.9,136.4,129.2,129.1,126.1,125.9$, 76.9, 73.5, 63.0, 62.8, 53.7, 50.9, 40.1, 39.9, 33.2, 30.7, 20.7, 20.7; MS $(m / z): 244.1\left(\mathrm{M}^{+}+\mathrm{Na}, 100\right), 245.2$ $\left(\mathrm{M}^{+}+1+\mathrm{Na}, 28\right)$; Anal. calcd. for $\mathrm{C}_{12} \mathrm{H}_{15} \mathrm{CNO}_{3}(\%)$ : C, 65.14; H, 6.83; N, 6.33; Found: C, 65.10; H, 6.88; $\mathrm{N}, 6.29$.

2.3d 6-(Hydroxymethyl)-4-(4-methoxyphenyl)-1, 3-oxazinan-2-one (4d): Yield: 70\% (light brown solid); mp: $132-135^{\circ} \mathrm{C}$; IR $\left(\mathrm{KBr}, \mathrm{cm}^{-1}\right): 3450,3254,3097,2930$, 1655, 1587, 1508, 1459, 1351, 1297, 1253, 1081, 1023, 767; ${ }^{1} \mathrm{H}$ NMR (DMSO, $\left.400 \mathrm{MHz}\right): \delta 7.65(\mathrm{~m}, 1 \mathrm{H})$, $7.20(\mathrm{~d}, J=12 \mathrm{~Hz}, 2 \mathrm{H}), 6.94(\mathrm{~d}, J=8 \mathrm{~Hz}, 2 \mathrm{H})$, 
4.93 (br, m. 1H), 4.62 (br, m, 1H), 3.99-3.96 (m, 1H), $3.74(\mathrm{~s}, 3 \mathrm{H}), 3.45-3.44(\mathrm{~m}, 1 \mathrm{H}), 2.08-2.00(\mathrm{~m}, 1 \mathrm{H})$, 1.86-1.82 (br, m, 1H); ${ }^{13} \mathrm{C}$ NMR (DMSO, $100 \mathrm{MHz}$ ): $\delta$ 158.4, 153.0, 135.1, 127.1, 113.3, 73.4, 62.8, 55.1, 50.5, 30.8; MS $(m / z) 260.8\left(\mathbf{M}^{+}+\mathrm{Na}, 100\right)$; Anal. calcd. for $\mathrm{C}_{12} \mathrm{H}_{15} \mathrm{NO}_{4}(\%)$ : C, 60.75; H, 6.37; N, 5.90; Found: C, $60.71 ; \mathrm{H}, 6.34 ; \mathrm{N}, 5.85$.

2.3e 6-(Hydroxymethyl)-4-(naphthalene-6-yl)-1, 3-oxazinan-2-one $(3 \boldsymbol{e}+4 \boldsymbol{e})$ : Yield: $76 \%$ (colourless solid); mp: $170-172^{\circ} \mathrm{C}$; IR $\left(\mathrm{KBr}, \mathrm{cm}^{-1}\right): 3443,3249,3129$, 2931, 1702, 1507, 1425, 1336, 1155, 1073, 762; ${ }^{1} \mathrm{H}$ NMR (DMSO, $400 \mathrm{MHz}): \delta 7.95-7.46(\mathrm{~m}, 16 \mathrm{H}), 5.00$ 4.93 (br, m, 2H), 4.85 (br, m, 1H), 4.77-4.73 (m, 1H), 4.41-4.38 (m, 1H), 4.03-4.01 (m, 1H), 3.53-3.46 (m, 2H), 2.20-2.12 (m, 2H), 2.01-1.98 (m, 1H), 1.74-1.63 (m, $1 \mathrm{H}) ;{ }^{13} \mathrm{C}$ NMR (DMSO, $\left.75 \mathrm{MHz}\right): \delta 153.3,153.1$, $140.9,139.7,132.8,132.7,132.5,132.2,128.2$, 127.8, $127.7,127.5,127.5,126.4,126.3,126.0,124.7,124.6$, 124.5, 125.4, 76.8, 73.3, 62.9, 62.7, 53.9, 51.2, 33.0, 30.3; MS $(m / z): 280.1\left(\mathrm{M}^{+}+\mathrm{Na}, 100\right), 279.2(12)$, 264.0 (5); Anal. calcd. for $\mathrm{C}_{15} \mathrm{H}_{15} \mathrm{NO}_{3}(\%)$ : C, 70.02; $\mathrm{H}$, 5.88; N, 5.44; Found: C, 69.98; H, 5.91; N, 5.41.

$2.3 f$ 4-(4-Flurophenyl)-6-(hydroxymethyl)-1, 3-oxazinan2-one (4f): Yield:70\% (colourless solid); mp: 102$105^{\circ} \mathrm{C}$; IR $\left(\mathrm{KBr}, \mathrm{cm}^{-1}\right)$ : 3298, 2919, 1708, 1603, 1509, 1433, 1351, 1293, 1156, 1052, 767; ${ }^{1} \mathrm{H}$ NMR (DMSO, $400 \mathrm{MHz}): \delta 7.71(\mathrm{~m}, 1 \mathrm{H}), 7.36-7.32(\mathrm{~m}, 2 \mathrm{H}), 7.23$ $7.19(\mathrm{~m}, 2 \mathrm{H}), 4.97$ (br, 1H), 4.70-4.67 (m, 1H), 3.99$3.93(\mathrm{~m}, 1 \mathrm{H}), 3.46-3.45(\mathrm{~m}, 1 \mathrm{H}), 2.11-2.04(\mathrm{~m}, 1 \mathrm{H})$, 1.89-1.85 (m, 1H); ${ }^{13} \mathrm{CNMR}$ (DMSO, $\left.100 \mathrm{MHz}\right): \delta$ $162.5,153.0,139.4,128.0,127.9,115.2,115.0,73.4$, 62.7, 50.5, 30.6; $\mathrm{MS}(\mathrm{m} / z): 248.1\left(\mathrm{M}^{+}+\mathrm{Na}, 100\right)$; Anal. calcd. for $\mathrm{C}_{11} \mathrm{H}_{12} \mathrm{FNO}_{3}(\%)$ : C, 58.66; H, 5.37; N, 6.22; Found: C, 58.63; H, 5.34; N, 6.19.

2.3g 6-(Hydroxymethyl)-4-(2-phenylethyl)-1, 3-oxazinan2-one $(3 g+4 g)$ : Yield: $85 \%$ (yellowish oily liquid); IR $\left(\mathrm{KBr}, \mathrm{cm}^{-1}\right): 3445,1701,1593,1351,1455,1335$,
1092, 677; ${ }^{1} \mathrm{H}$ NMR (DMSO, $\left.400 \mathrm{MHz}\right): \delta 7.38-7.37$ $(\mathrm{m}, 1 \mathrm{H}), 7.30(\mathrm{~m}, 1 \mathrm{H}), 7.28-7.15(\mathrm{~m}, 10 \mathrm{H}), 4.98$ (br, 2H), 4.30-4.24 (m, 1H), 4.19-4.13 (m, 1H), 3.49$3.48(\mathrm{~m}, 4 \mathrm{H}), 2.67-2.54(\mathrm{~m}, 4 \mathrm{H}), 2.04-2.00(\mathrm{~m}, 1 \mathrm{H})$, $1.87-1.74(\mathrm{~m}, 4 \mathrm{H}), 1.72-1.59(\mathrm{~m}, 2 \mathrm{H}), 1.41-1.32$ $(\mathrm{m}, 1 \mathrm{H}) ;{ }^{13} \mathrm{C}$ NMR (DMSO, $\left.100 \mathrm{MHz}\right): \delta 153.2,152.9$, 141.6, 141.6, 128.3, 128.3, 128.3, 128.3, 125.8, 125.8, 76.9, 74.2, 63.1, 62.8, 49.4, 47.1, 37.9, 37.4, 31.1, 30.3, 29.1, 26.8; $\mathrm{MS}(\mathrm{m} / \mathrm{z}): 258.0\left(\mathrm{M}^{+}+\mathrm{Na}, 21\right), 241.6$ (41); Anal. calcd. for $\mathrm{C}_{13} \mathrm{H}_{17} \mathrm{NO}_{3}(\%)$ : C, 66.36; H, 7.28; N, 5.95; Found: C, 66.38; H, 7.31; N, 5.92.

2.3h 6-(Hydroxymethyl)-4-(2-methoxyphenyl)-1, 3-oxazinan-2-one $(\mathbf{3 h}+\mathbf{4 h})$ : Yield: $71 \%$ (colourless solid); $\mathrm{mp}: 154-155^{\circ} \mathrm{C}$; IR $\left(\mathrm{KBr}, \mathrm{cm}^{-1}\right)$ : $3373,3244,3107$, 2939, 1690, 1597, 1344, 1294, 1246, 1106, 1081, 759; ${ }^{1} \mathrm{H}$ NMR (DMSO, $\left.300 \mathrm{MHz}\right): \delta$ 7.55-7.54 (m, 1H), 7.43 (br, s, 1H), 7.32-7.18 (m, 4H), 7.04-6.96 (m, 4H), 4.93-4.87 (m, 2H), 4.85-4.82 (m, 2H), 4.34-4.30 (m, $1 \mathrm{H}), 3.94-3.87(\mathrm{~m}, 1 \mathrm{H}), 3.81(\mathrm{~s}, 3 \mathrm{H}), 3.79(\mathrm{~s}, 3 \mathrm{H})$, 3.49-3.48 (m, 2H), $2.15(\mathrm{dd}, J=15 \mathrm{~Hz}, 6 \mathrm{~Hz}, 1 \mathrm{H})$, 2.05-1.96 (m, 1H), 1.88-1.83 (br, m, 1H), 1.51-1.39 (m, 1H); ${ }^{13} \mathrm{C}$ NMR (DMSO, $75 \mathrm{MHz}$ ): $\delta 156.1,155.5$, $153.7,153.4,130.5,129.8,128.5,128.5,126.6,125.9$, $120.6,120.1,110.9,110.9,76.7,73.5,62.9,55.5,55.4$, 47.8, 46.7, 31.0, 28.3; MS $(m / z): 260.8\left(\mathrm{M}^{+}+\mathrm{Na}\right.$, 100); Anal. calcd. for $\mathrm{C}_{12} \mathrm{H}_{15} \mathrm{NO}_{4}(\%)$ : C, 60.75; $\mathrm{H}$, 6.37; N, 5.90; Found: C, 60.78; H, 6.40; N, 5.92.

\section{Results and discussion}

Although a number of methods are available for the synthesis of 1,3-oxazinan-2-one, development of new simple and efficient synthetic pathways for preparation of such compounds are quite interesting for a synthetic chemist. Due to the wide biological activity of oxazinanones, synthesis of new analogues and a study of their biological properties is essential. We report the synthesis of some new analogues of 1,3oxazinan-2-ones starting from homoallylic carbamate (scheme 1).

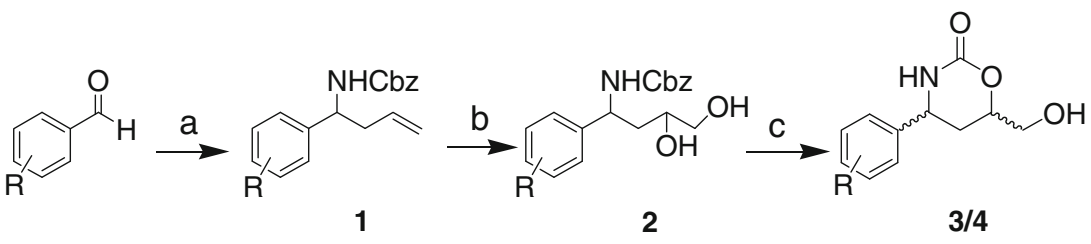

$\mathrm{R}=\mathrm{Cl}, \mathrm{Br}, \mathrm{F}, \mathrm{Me}$, OMe, etc.

Reagent and condition: (a) Allyltrimethylsilane, benzyl carbamate, $\mathrm{I}_{2}, \mathrm{CH}_{3} \mathrm{CN}$, rt; (b) $\mathrm{K}_{2} \mathrm{OsO}_{2}(\mathrm{OH})_{4}, \mathrm{~K}_{3} \mathrm{Fe}(\mathrm{CN})_{6}, \mathrm{~K}_{2} \mathrm{CO}_{3}$, pyridine, $\mathrm{tBuOH}: \mathrm{H}_{2} \mathrm{O}$ (1:1); (c) $\mathrm{NaH}$, THF, $0^{\circ} \mathrm{C}$

Scheme 1. Synthetic pathway for 1,3-oxazinan-2-one. 
We have synthesized some new 4,6-disubstituted 1,3-oxazinan-2-one using homoallyl amine via Sharpless dihydroxylation and cyclization strategy. In our approach, we have used Cbz-protected homoallyl amine $\mathbf{1}$ as the staring material which was prepared by a threecomponent condensation reaction of aromatic aldehyde, benzyl carbamate and allyltrimethylsilane, developed in our laboratory. ${ }^{42}$ Initially, the synthesis (scheme 1) was carried out with homoallylic carbamate 1a, prepared from 4-chloro benzaldehyde. The compound 1a was prepared by adding benzyl carbamate (1.05 equiv) and allyltrimethylsilane (1 equiv) to a solution of 4-chloro benzaldehyde (1 equiv) and iodine $(10 \mathrm{~mol} \%)$ in acetonitrile at room temperature. ${ }^{42 a}$ The protected homoallyl amine 1a was then subjected to Sharpless dihydroxylation. ${ }^{43}$ The reaction was carried out using standard dihydroxylation reaction condition in presence of a small

Table 1. Synthesis of 4,6-disubstituted 1,3-oxazinan-2-one derivatives.

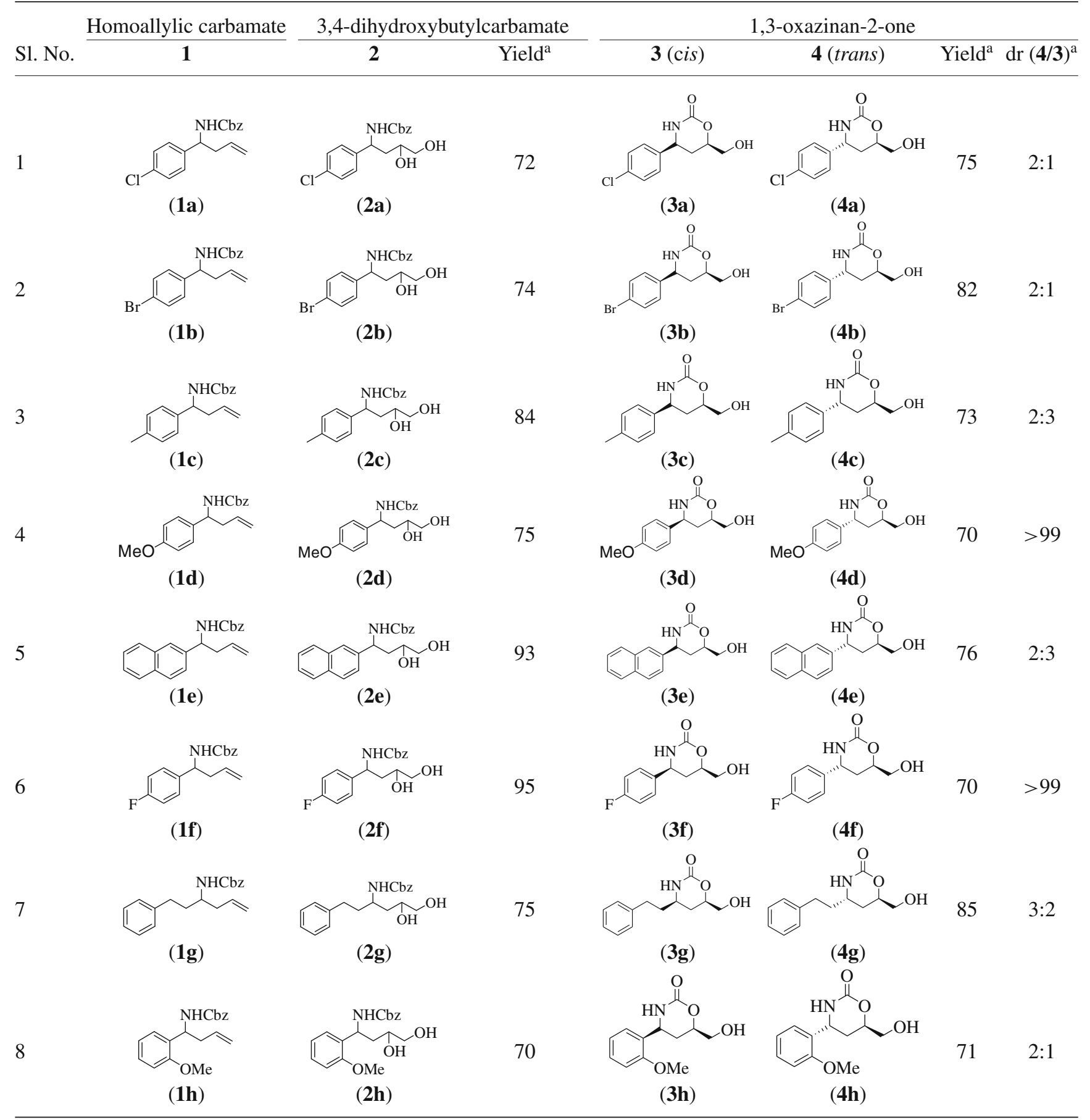

aisolated yield after chromatographic purifications 
amount of pyridine to produce corresponding 3,4dihydroxybutylcarbamate derivative $\mathbf{2 a}$. The product was found to be an inseparable mixture of two diastereomers. The 3,4-dihydroxybutylcarbamate derivative 2a was then treated with $\mathrm{NaH}$ in THF at $0^{\circ} \mathrm{C}$. This baseinduced intramolecular cyclization reaction ${ }^{44}$ resulted in the formation of a diastereomeric mixture of 6membered 1, 3-oxazinan-2-one (3a $+\mathbf{4 a})$ in $75 \%$ yield. The nuclear magnetic resonance (NMR) studies indicate the presence of two diastereomers in the final product. In this case also, it was not possible to separate the diastereomers using column chromatography and was isolated as a mixture of cis and trans products. Relative stereochemistry of the products has been assigned by comparison of the chemical shifts of $-\mathbf{C H}_{2} \mathrm{OH}$ protons with data on similar skeleton already reported in literature. ${ }^{30}$ Diastereomeric ratio (cis : trans) was calculated using ${ }^{1} \mathrm{H}$ NMR and found to be $1: 2$. In the ${ }^{1} \mathrm{H}$ NMR spectrum, one of the $-\mathbf{C H}_{2} \mathrm{OH}$ protons appear as two different multiplets at 4.34-4.30 $\delta$ (for cis isomer) and 3.97-3.92 $\delta$ (for trans isomer) with a ratio of 1:2. The other proton in the same group, appears at 3.52$3.44 \delta$ as multiplet (for both diastereomers). In a similar way, one proton of 5- $\mathbf{C H}_{2}$ group appears as a multiplet at $1.89-1.86 \delta$ (trans isomer), while the same proton in the other diastereomer appears at $1.59-1.50 \delta$ (cis isomer) with a ratio of 2:1 (trans:cis). The other proton for both diastereomers comes at 2.13-2.05 $\delta$ as multiplet.

After optimizing the synthetic strategy, we have expanded the scope of the process using a number of homoallylic carbamates prepared with different aromatic aldehydes. Results are presented in table 1.

In general, this strategy works well for a variety of aldehydes to produce the corresponding 4,6disubstituted-1,3-oxazinan-2-one derivatives in high yield. All the products were characterized through IR, NMR and mass apectral data. Integration ratio of proton in ${ }^{1} \mathrm{H}$ NMR spectra of the cyclic carbamate reveals the formation of diastereomers in a 1:2 ratio (cis: trans) for the entries $(1,2,8)$; while $1: 1.5$ (cis : trans) selectivity was observed for entry 7 . However for the entries 3 and 5, the cis-isomer was found to be dominant over the trans-isomer with diasteromeric ratio 1.5:1 (cis : trans). Interestingly for the entries 4 and 6 (table 1), only transisomers $\mathbf{4 d}$ and $\mathbf{4 f}$, respectively, were formed predominantly. This may be due to strong resonance effect of substituents on the aromatic ring.

\section{Conclusion}

In conclusion, we have developed an efficient synthetic strategy for the synthesis of 4,6-disubstituted 1,3- oxazinan-2-one derivatives starting from a homoallylic amine. Although, in most of the cases, a mixture of diostereomers is formed, 6-(hydroxymethyl)-4-(4methoxyphenyl)-1,3-oxazinan-2-one and 4-(4-flurophenyl)-6-(hydroxymethyl)-1,3-oxazinan-2-one were formed predominantly with only trans diastereomer.

\section{Supplementary information}

For ${ }^{1} \mathrm{H}$ NMR and ${ }^{13} \mathrm{NMR}$ spectra as supporting information see www.ias.ac.in/chemschi: website.

\section{Acknowledgements}

Financial support from the Council of Scientific and Industrial Research, New Delhi (Grant no. 01(1933)/ 04/EMR-II) is gratefully acknowledged. AB thanks DST-JBNSTS for providing a visiting studentship to work in the Department of Organic Chemistry, Indian Institute of Science (IISc), Bangalore. We also thank Prof. K R Prasad (IISc, Bangalore) for extending his laboratory facilities during the initial stage of this study.

\section{References}

1. (a) Wang G, Ella-Menye J R and Sharma V 2006 Bioorg. Med. Chem. Lett. 16 2177; (b) Ullrich T, Baumann K, Welzenbach K, Schmutz S, Camenisch, G, Meingassner J G and Weitz-Schmidt G 2004 Bioorg. Med. Chem. Lett. 14 2483; (c) Zanatta N, Borchhardt D M, Alves S H, Coelho H S, Squizani A M C, Marchi T M, Bonacorso H G and Martins M A P 2006 Bioorg. Med. Chem. Lett. 14 3174; (d) Zhang P, Terefenko E A, Fensome A, Wrobel J, Winneker R and Zhang Z 2003 Bioorg. Med. Chem. Lett. 13 1313; (e) Roller S G, Dieckhaus C M, Santos W L, Sofia R D and MacDonald T L 2002 Chem. Res. Toxicol. 15815

2. Corey E J and Cheng X-M 1989 The logic of chemical synthesis (New York: John Wiley \& Sons) p. 423

3. Gormley Jr G, Chan Y Y and Fried J 1980 J. Org. Chem. 451447

4. (a) Larson G M, Schaneberg B T and Sneden A T 1999 J. Nat. Prod. 62 361; (b) Cassady J M, Chan K K, Floss H G and Leistner E 2004 Chem. Pharm. Bull. 52 1; (c) Widdison W C, Wilhelm S D, Cavanagh E E, Whiteman K R, Leece B A, Kovtun Y, Goldmacher V S, Xie H, Steeves R M, Lutz R J, Zhao R, Wang L, Blattler A and Chari R V J 2006 J. Med. Chem. 494392

5. (a) Wang G 2008 Anti-Infect. Agents Med. Chem. 7 32; (b) Wang G, Ella-Menye J-R and Sharma V 2006 Bioorg. Med. Chem. Lett. 162177

6. Ullrich T, Baumann K, Welzenbach K, Schmutz S, Camenisch G, Meingassner J G and Weitz-Schmidt G 2004 Bioorg. Med. Chem. Lett. 142483

7. Jin F 2000 Confalone P N PCT Int. Appl. WO0000481, 119; Chem. Abstr. 13278560 
8. Engel J, Emig P, Nickel B and Szelenyi I 1990 Ger. Often DE 3.915.184: Chem. Abstr. 1122352861

9. Rajadhyaksha V J 1990 PCT Int. Appl. WO 90,00407: Chem. Abstr. 11359177

10. Testa E, Fontanella L, Cristiani G and Gallo G $1959 \mathrm{~J}$. Org. Chem. 241928

11. Franran C P, Douzon C, Raynaud G M and Serganl M Y 1975 U.S. 3.821.215: Chem. Abstr. 8249951

12. Yamana K, Suzuki N and Takahama A 2002 Ine, S. Jpn. Kokai Tokkyo Koho JP 2002179572; Chem. Abstr. 137 57555

13. Hirama M, Shigemoto T and Ito S $1987 \mathrm{~J}$. Org. Chem. 523342

14. Wang Y-F, Izawa T, Kobayashi S and Ohno M $1982 \mathrm{~J}$. Am. Chem. Soc. 1046465

15. Woodward R B, Logusch E, Nambiar K P, Sakan K, Ward D E, Au-Yeung B-W, Balaram P, Browne L J, Card P J, Chen C H, Chenevert R B, Fliri A and Frobel K et al. 1981 J. Am. Chem. Soc. 1033213

16. (a) Hirama M, Shigemoto T, Yamazaki Y and Ito S 1985 J. Am. Chem. Soc. 107 1797; (b) González-Rosende M E, Jordá-Gregori J M, Sepúlveda-Arques J and Orena M 2004 Tetrahedron: Asymm. 15 419; (c) Bongini A, Cardillo G and Orena M 1988 Chem. Lett. 87

17. (a) Abbas T R, Cadogan J I G, Doyle A A, Gosney I, Hodgson P K G, Howells G E, Hulme A N, Parsons S and Sadler I H 1997 Tetrahedron Lett. 38 4917; (b) Banks M R, Cadogan J I G, Gosney I, Gould R O, Hodgson K G, and McDougall D 1998 Tetrahedron 54 9765; (c) Osa Y, Hikima Y, Sato Y, Takino K, Ida Y, Hirono S and Nagase H $2005 \mathrm{~J}$. Org. Chem. 70 5737; (d) Takahata H, Saito Y and Ichinose M 2006 Org. Biomol. Chem. 4 1587; (e) Wang C and Tunge J A 2006 Org. Lett. 8 3211; (f) Perch N S and Widenhoefer R A 1999 J. Am. Chem. Soc. 121 6960; (g) Perry M C, Powell M T, Cui X, Hou D-R, Reibenspies J H and Burgess K $2003 \mathrm{~J}$. Am. Chem. Soc. 125 113

18. Takiguchi T, Iwaki T, Tokanou G, Kosaka $\mathrm{Y}$ and Nakamura S JP9151179 1997; Chem. Abstr. 127 42427

19. Lohray B B, Baskaran S, Reddy B Y and Rao K S 1998 Tetrahedron Lett. 396555

20. Ross B C 1977 U.S. Patent 4107435, Chem. Abstr. 88 7304

21. Fujita M, Kitagawa O, Suzuki T and Taguchi T 1997 J. Org. Chem. 627330

22. Jorda-Gregori J M, Gonzalez-Rosende M E, CavaMontesinos P, Sepulveda-Arques J, Galeazzi R and Orena M 2000 Tetrahedron: Asymm. 113769

23. Muehlstaedt M, Meusinger R, Olk B, Weber L and Widera R 1986 J. Prakt. Chem. 328309
24. Davies S G, Haggitt J R, Ichihara O, Kelly R J, Leech M A, Price Mortimer A J, Roberts P M and Smith A D 2004 Org. Biomol. Chem. 22630

25. Takahata H, Ouchi H, Ichinose M and Nemoto H 2002 Org. Lett. 43459

26. Jung J-W, Shin D-Y, Seo S-Y, Kim S-H, Paek S-M, Jung J-K and Suh Y-G 2005 Tetrahedron Lett. 46573

27. Puigbó G, Diaba F and Bonjoch J 2003 Tetrahedron 59 2657

28. Davies S G and Ichihara O 1999 Tetrahedron Lett. 40 9313

29. (a) Yamamoto $\mathrm{Y}$, Komatsu $\mathrm{T}$ and Muruyama $\mathrm{K} 1985$ J. Org. Chem. 50 3115; (b) Yamamoto Y, Nishii S, Muruyama K, Komatsu T and Ito W $1986 \mathrm{~J}$. Am. Chem. Soc. 108 7778; (c) David M, Dhimane H, VanucciBacqué C, Lhommet G 1999 J. Org. Chem. 64 8402; (d) Jones A D, Knight D W and Hibbs D E $2001 \mathrm{~J}$. Chem. Soc. Perkin Trans. 11182

30. Mangelimckx S, Nural Y, Dondas H A, Denolf B, Sillanpää R and Kimpe N D 2010 Tetrahedron 66 4115

31. Kemp S J, Bao J G and Pedersen S F 1996 J. Org. Chem. 617162

32. Sonoda N, Yamamoto G, Natsukawa K, Kondo K and Murai S 1975 Tetrahedron Lett. 161969

33. Walsh P J, Bennani Y L and Sharpless K B 1993 Tetrahedron Lett. 345545

34. Donohoe T J, Bataille C R R, Gattrell W, Kloesges J and Rossignol E 2007 Org. Lett. 91725

35. Hilborn J W, Lu Z-H, Jurgens A R, Fang Q K, Byers P, Wald S A and Senanayake C H 2001 Tetrahedron Lett. 428919

36. Hirama M, Nishizaki I, Shigemoto T and Ito S $1986 \mathrm{~J}$. Chem. Soc. Chem. Commun. 393

37. Ella-Menye J-R, Sharma V and Wang G 2005 J. Org. Chem. 70463

38. Davies S G, Garner A C, Roberts P M, Smith A D, Sweet M J and Thomson J E 2006 Org. Biomol. Chem. 4 2753

39. Ella-Menye J-R and Wang G 2007 Tetrahedron 63 10034

40. Murakami M, Suginome M, Fujimoto K, Nakamura H, Andersson P G and Ito Y 1993 J. Am. Chem. Soc. 115 6487

41. Jung J-C and Avery M A 2006 Tetrahedron Lett. 47 7969

42. (a) Phukan P 2004 J. Org. Chem. 69 4005; (b) Kalita H $\mathrm{R}$ and Phukan P 2005 Synth. Commun. 35485

43. Kolb H C, van Nieuwenhze M S and Sharpless K B 1994 Chem. Rev. 942483

44. Ager D J, Prakash I and Schaad D R 1996 Chem. Rev. 96835 